A. L. Shidlich (Institute of Mathematics of National Academy of Sciences of Ukraine, Kyiv, Ukraine)

S. O. Chaichenko (Donbas State Pedagogical University, Slavyansk, Ukraine)

\title{
Approximative properties of diagonal operators in Orlicz spaces
}

We obtain the exact values of some important approximative quantities (such as, the best approximation, the basis width, Kolmogorov's width and the best n-term approximation) of certain sets of images of the diagonal operators in the Orlicz sequence spaces $l_{M}$.

1. Introduction. Let $M(t), t \geq 0$, be an Orlicz function, that is a non-decreasing convex down function such that $M(0)=0$ and $M(t) \rightarrow \infty$ as $t \rightarrow \infty$. Orlicz sequence space $l_{M}$ [1], defined by the function $M(t)$, is the linear space of all sequences $x=\left\{x_{k}\right\}_{k=1}^{\infty}$ of real numbers such that $\sum_{k} M\left(x_{k}\right)<\infty$. Equipped with the norm

$$
\|\mathbf{x}\|_{l_{M}}:=\inf \left\{\alpha>0: \sum_{k=1}^{\infty} M\left(\left|x_{k}\right| / \alpha\right) \leq 1\right\}
$$

it is a Banach space.

An Orlicz function $M(t)$ is said to satisfy the $\Delta_{2}$-condition, if for all $t \geq 0$, the inequality $M(2 t) \leq C M(t)$ holds, where $C>0$ is a certain constant.

Note that in the case, where $M(t)=t^{p}, p \geq 1$, the spaces $l_{M}$ coincide with the usual spaces $l_{p}$ with the norm

$$
\|\mathbf{x}\|_{l_{p}}=\left(\sum_{k=1}^{\infty}\left|x_{k}\right|^{p}\right)^{1 / p}
$$

If the function $M(t)$ satisfies $\Delta_{2}$-condition, then the following equality is true (see [2, Proposition 1]):

$$
l_{M}=\left\{\mathbf{x}=\left\{x_{k}\right\}_{k=1}^{\infty}: \quad \sum_{k=1}^{\infty} M\left(\left|x_{k}\right| / \alpha\right)<\infty \quad \forall \alpha>0\right\},
$$

and the system of vectors $\left(e_{i}\right)_{i=1}^{\infty}$ (where $e_{i}=\left\{e_{i k}\right\}_{k=1}^{\infty}, e_{i k}=0$ if $k \neq i$ and $e_{i i}=1$ ) form a basis in this space.

In 1932, W. Orlicz [3] considered the spaces $L_{M}$ of the measurable functions $f$ such that $\int M(|f(t)|) d t<\infty$ and investigated some properties of these spaces, assuming that the function $M$ satisfies $\Delta_{2}$-condition. The spaces $L_{M}$ are a natural generalization of the Lebesgue spaces $L_{p}$ and they are called Orlicz spaces. Further, there were introduced Orlicz spaces of the functions defined on the sets of the infinite measure, as well as on the sets with no continuous measure (see, for example, monographs [4, 5]). In the particular case, when the definition set consists of the sequence of points, each of which has a unit measure, the spaces $L_{M}$ are Orlicz sequence spaces $l_{M}$ and they are a generalization of well-known spaces $l_{p}$. The spaces $l_{M}$ were introduced by W. Orlicz in the paper [1] and subsequently studied by many authors (see, for example, [2, 66 10]). 
In this paper, we consider the approximative properties of the diagonal operators in Orlicz sequence spaces. We obtain the exact values of some important approximative quantities (such as, the best approximation, the basis width, Kolmogorov's width, the best $n$-term approximation) of certain sets of images of the diagonal operators in the spaces $l_{M}$. In particular, the obtained results are a generalization of similar results for the spaces $l_{p}\left(l_{p}^{d}\right)$ 11 14.

2. Best approximations and basis widths. Let $\lambda=\left\{\lambda_{k}\right\}_{k=1}^{\infty}$ be an arbitrary sequence of the positive numbers satisfying the condition

$$
\lim _{k \rightarrow \infty} \lambda_{k}=0
$$

and let $T: x=\left\{x_{k}\right\}_{k=1}^{\infty} \rightarrow T x=\left\{\lambda_{k} x_{k}\right\}_{k=1}^{\infty}$ be a diagonal operator defined on the space $l_{M}$.

Further, let $M(t)$ and $N(t)$ be two arbitrary Orlicz functions, let $l_{M}$ and $l_{N}$ be the Orlicz spaces corresponding to these functions. Let also $B l_{M}$ be the unit ball of the space $l_{M}$. For any fixed collection $\gamma_{n}$ of $n, n \in \mathbb{N}$, positive integers, consider the quantity

$$
E_{\gamma_{n}}\left(T: l_{M} \rightarrow l_{N}\right):=E_{\gamma_{n}}\left(T\left(B l_{M}\right), l_{N}\right)=\sup _{x \in B l_{M}} E_{\gamma_{n}}\left(T x, l_{N}\right)=\sup _{x \in B l_{M}} \inf _{a_{i}}\left\|T x-P_{\gamma_{n}}\right\|_{l_{N}}
$$

of the best approximation in the space $l_{N}$ of the set $T\left(B l_{M}\right)$ by all possible $n$-term polynomial $P_{\gamma_{n}}=\sum_{i \in \gamma_{n}} a_{i} e_{i}$, corresponding to the set $\gamma_{n}$, where $a_{i}$ are arbitrary real numbers.

Note that if

$$
0<N(t) \leq M(t), \quad t \in[0,1]
$$

and the sequence $\lambda$ satisfies condition (4), then for any $x \in B l_{M}$, we have $T x \in l_{N}$. Hence, in such conditions, the quantity $E_{\gamma_{n}}\left(T: l_{M} \rightarrow l_{N}\right)$ is well defined.

Indeed, in this case, putting $\lambda^{*}:=\max _{k \in \mathbb{N}} \lambda_{k}$, in view of nonincreasing the functions $M(t)$ and $N(t)$, for any $x \in B l_{M}$, we have

$$
\sum_{k=1}^{\infty} N\left(\frac{\lambda_{k}\left|x_{k}\right|}{\lambda^{*}}\right) \leq \sum_{k=1}^{\infty} N\left(\left|x_{k}\right|\right) \leq \sum_{k=1}^{\infty} M\left(\left|x_{k}\right|\right) \leq \sum_{k=1}^{\infty} M\left(\frac{\left|x_{k}\right|}{\| x||_{l_{M}}}\right) \leq 1 .
$$

This yields that $\|T x\|_{l_{N}} \leq \lambda^{*}<\infty$ and $T x \in l_{N}$.

Theorem 1. Assume that $M(t)$ and $N(t)$ are arbitrary Orlicz functions, satisfying the relations (5) and

$$
\inf \{\alpha>0: M(1 / \alpha) \leq 1\}=\inf \{\alpha>0: N(1 / \alpha) \leq 1\}
$$

Let $\lambda=\left\{\lambda_{k}\right\}_{k=1}^{\infty}$ be an arbitrary sequence of the positive numbers for which condition (44) holds. Then for any collection $\gamma_{n}$ of $n, n \in \mathbb{N}$, positive integers, the following equality is true:

$$
E_{\gamma_{n}}\left(T: l_{M} \rightarrow l_{N}\right)=\max _{k \notin \gamma_{n}} \lambda_{k}
$$


Proof. Since for any $x \in l_{N}, \alpha>0$ and $a_{i} \in \mathbb{R}$,

$$
\sum_{k \in \gamma_{n}} N\left(\left|x_{k}-a_{i}\right| / \alpha\right)+\sum_{k \notin \gamma_{n}} N\left(\left|x_{k}\right| / \alpha\right) \geq \sum_{k \notin \gamma_{n}} N\left(\left|x_{k}\right| / \alpha\right)
$$

then for any $x \in l_{N}$, we have

$$
E_{\gamma_{n}}\left(x, l_{N}\right)=\mathscr{E}_{\gamma_{n}}\left(x, l_{N}\right):=\left\|x-S_{\gamma_{n}}(x)\right\|_{l_{N}}=\inf \left\{\alpha>0: \sum_{k \notin \gamma_{n}} N\left(\left|x_{k}\right| / \alpha\right) \leq 1\right\}
$$

where $S_{\gamma_{n}}(x)=\sum_{k \in \gamma_{n}} x_{k} e_{k}$.

Put $\lambda_{k^{*}}=\lambda_{k^{*}}\left(\gamma_{n}\right)=\max _{k \notin \gamma_{n}} \lambda_{k}$. For any $x \in B l_{M}$, we get

$$
\sum_{k \notin \gamma_{n}} N\left(\frac{\lambda_{k}\left|x_{k}\right|}{\lambda_{k^{*}}}\right) \leq \sum_{k \notin \gamma_{n}} N\left(\left|x_{k}\right|\right) \leq \sum_{k \notin \gamma_{n}} M\left(\left|x_{k}\right|\right) \leq \sum_{k \notin \gamma_{n}} M\left(\frac{\left|x_{k}\right|}{\| x||_{l_{M}}}\right) \leq 1
$$

This yields

$$
E_{\gamma_{n}}\left(T: l_{M} \rightarrow l_{N}\right) \leq \lambda_{k^{*}}=\max _{k \notin \gamma_{n}} \lambda_{k}
$$

On the other hand, consider the element $x^{*}=e_{k^{*}} /\left\|e_{k^{*}}\right\|_{l_{M}}$. By virtue of ([6]), we have

$$
\left\|e_{k^{*}}\right\|_{l_{M}}=\inf \{\alpha>0: M(1 / \alpha) \leq 1\}=\inf \{\alpha>0: N(1 / \alpha) \leq 1\}=\left\|e_{k^{*}}\right\|_{l_{N}}
$$

Therefore $\left\|x^{*}\right\|_{l_{M}}=\left\|x^{*}\right\|_{l_{N}}=1$ and $x^{*} \in B l_{M}$. It follows that

$$
E_{\gamma_{n}}\left(T x^{*}, l_{N}\right)=\inf \left\{\alpha>0: N\left(\frac{\lambda_{k^{*}}}{\alpha\left\|e_{k^{*}}\right\|_{l_{M}}}\right) \leq 1\right\}=\inf \left\{\alpha>0: N\left(\frac{\lambda_{k^{*}}}{\alpha\left\|e_{k^{*}}\right\|_{l_{N}}}\right) \leq 1\right\}=\lambda_{k^{*}} .
$$

Thus, equality (77) is really true.

Note that relation (6) holds, in particular, when $M(1)=N(1)=1$.

Considering the lower bounds of both sides of equality (7) over all possible collections $\gamma_{n}$ of $n$ natural numbers, we conclude that the least lower bound of the right-hand side of (7) is realized by the collection $\gamma_{n}^{*}$ defined by the relation

$$
\gamma_{n}^{*}=\left\{i_{k} \in \mathbb{N}: \quad \lambda_{i_{k}}=\bar{\lambda}_{k}, k=1,2, \ldots, n\right\}, \quad n=1,2, \ldots
$$

where $\bar{\lambda}=\left\{\bar{\lambda}_{k}\right\}_{k=1}^{\infty}$ is nondecreasing rearrangement of the numbers $\lambda_{k}$ and $\max _{k \notin \gamma_{n}^{*}} \lambda_{k}=\bar{\lambda}_{n+1}$.

Thus, the following statement is true:

Corollary 1. Assume that $M(t)$ and $N(t)$ are arbitrary Orlicz functions, satisfying the relations (5) and (6) . Let $\lambda=\left\{\lambda_{k}\right\}_{k=1}^{\infty}$ be an arbitrary sequence of the positive numbers for which the condition (4) holds. Then for any $n \in \mathbb{N}$,

$$
D_{n}\left(T: l_{M} \rightarrow l_{N}\right)=D_{n}\left(T\left(B l_{M}\right), l_{N}\right):=\inf _{\gamma_{n}} E_{\gamma_{n}}\left(T: l_{M} \rightarrow l_{N}\right)=\bar{\lambda}_{n+1},
$$

where $\bar{\lambda}=\left\{\bar{\lambda}_{k}\right\}_{k=1}^{\infty}$ is the nonincreasing rearrangement of the numbers $\lambda_{k}$. 
The quantities $D_{n}\left(T: l_{M} \rightarrow l_{N}\right)=D_{n}\left(T\left(B l_{M}\right), l_{N}\right)$ are called the basis widths of order $n$ of the set $T\left(B l_{M}\right)$ in the space $l_{N}$.

Note that in the case, when $M(t)=t^{q}$ and $N(t)=t^{p}, 0<q \leq p$, i.e. when $l_{M}=l_{q}$ and $l_{N}=l_{p}$, the assertions of Theorem 1 and Corollary 1 follow from theorems 4.1 and 4.3 of the paper [13] respectively.

3. Kolmogorov's widths. The structure of the aggregates used for the approximation of the elements $x \in L_{M}$, is determined by the characteristic sequences $\varepsilon(\lambda), g_{n}(\lambda)$ and $\delta(\lambda)$. These characteristic sequences are defined as follows [13]:

Let $\lambda=\left\{\lambda_{k}\right\}_{k=1}^{\infty}$ be an arbitrary sequence of the positive numbers that satisfies condition (4). Then $\varepsilon(\lambda)=\varepsilon_{1}, \varepsilon_{2}, \ldots$ denotes the set of values of the quantities $\lambda_{k}$, enumerated in the nonincreasing order, $g(\lambda)=g_{1}, g_{2}, \ldots$ denotes the system of sets

$$
g_{n}=g_{n}^{\lambda}=\left\{k \in \mathbb{N}: \lambda_{k} \geq \varepsilon_{n}\right\}
$$

and $\delta(\lambda)=\delta_{1}, \delta_{2}, \ldots$ denotes the sequence of numbers $\delta_{n}=\left|g_{n}\right|$, where $\left|g_{n}\right|$ is the amount of numbers $k \in \mathbb{N}$ in the set $g_{n}$.

Taking into account condition (4), we can determine the sequences $\varepsilon(\lambda)$ and $g(\lambda)$ with the use of the following recurrence relations:

$$
\begin{gathered}
\varepsilon_{1}=\sup _{k \in \mathbb{N}} \lambda_{k}, \quad g_{1}=\left\{k \in \mathbb{N}: \lambda_{k}=\varepsilon_{1}\right\}, \\
\varepsilon_{n}=\sup _{k \bar{\epsilon} g_{n-1}} \lambda_{k}, \quad g_{n}=g_{n-1} \cup\left\{k \in \mathbb{N}: \lambda_{k}=\varepsilon_{n}\right\} .
\end{gathered}
$$

Note that, according to this definition, any number $n^{*} \in \mathbb{N}$ belongs to all the sets $g_{n}^{\lambda}$ for the sufficiently large $n$ and

$$
\lim _{k \rightarrow \infty} \delta_{k}=\infty
$$

In what follows, it is convenient to denote the empty set by $g_{0}=g_{0}^{\lambda}$ and assume that $\delta_{0}=0$.

Note also that if $\bar{\lambda}=\left\{\bar{\lambda}_{k}\right\}_{k=1}^{\infty}$ is the nonincreasing rearrangement of the numbers $\lambda_{k}, k=1,2, \ldots$, then the following equality is true:

$$
\bar{\lambda}_{k}=\varepsilon_{n} \quad \forall k \in\left(\delta_{n-1}, \delta_{n}\right], \quad n=1,2, \ldots
$$

Therefore, from Theorem 1, it is easily obtained the following corollary.

Corollary 2. Assume that $M(t)$ and $N(t)$ are arbitrary Orlicz functions, satisfying the relations (5) and (6) . Let $\lambda=\left\{\lambda_{k}\right\}_{k=1}^{\infty}$ be an arbitrary sequence of the positive numbers for which the condition (41) holds. Then for any $n \in \mathbb{N}$,

$$
E_{g_{n-1}^{\lambda}}\left(T: l_{M} \rightarrow l_{N}\right)=\mathscr{E}_{g_{n-1}^{\lambda}}\left(T: l_{M} \rightarrow l_{N}\right):=\sup _{x \in B l_{M}} \mathscr{E}_{g_{n-1}^{\lambda}}\left(T x, l_{N}\right)=\varepsilon_{n}
$$


where $\varepsilon_{n}$ is the $n$-th term of the characteristic sequence $\varepsilon(\lambda)$.

Remark 1. Note that if the sequence $\lambda=\left\{\lambda_{k}\right\}_{k=1}^{\infty}$ is a strictly decreasing, then for an arbitrary $n \in \mathbb{N}$, we have $\varepsilon_{n}(\lambda)=\lambda_{n}, g_{n}^{\lambda}=\{1,2, \ldots, n\}$ and $\delta_{n}(\lambda)=n$. Thus, for any $x \in l_{M}$, the quantities $E_{g_{n-1}^{\lambda}}\left(x, l_{M}\right)$ and $\mathscr{E}_{g_{n-1}^{\lambda}}\left(x, l_{M}\right)$ respectively have the forms

$$
E_{g_{n-1}^{\lambda}}\left(x, l_{M}\right)=E_{n-1}\left(x, l_{M}\right)=\inf _{a_{i}}\left\|x-\sum_{i=1}^{n-1} a_{i} e_{i}\right\|_{l_{M}}
$$

and

$$
\mathscr{E}_{g_{n-1}^{\lambda}}\left(x, l_{M}\right)=\mathscr{E}_{n-1}\left(x, l_{M}\right)=\left\|x-\sum_{i=1}^{n-1} x_{i} e_{i}\right\|_{l_{M}} .
$$

Further, assume that $X$ and $Y$ are the normed linear spaces, $B X$ is the closed unit ball of the space $X$, and $T: X \rightarrow Y$ is the bounded linear operator. The quantity

$$
d_{n}(T: X \rightarrow Y):=d_{n}(T(B X) ; Y)=\inf _{F_{n} \in \mathcal{F}_{n}} \sup _{x \in \mathfrak{B} X} \inf _{u \in F_{n}}\|x-u\|_{Y}
$$

is called Kolmogorov's widths of the set $T(B X)$ in the space $Y$. Here, $\mathcal{F}_{n}$ is the set of all the subspaces of the dimension $n \in \mathbb{N}$ of the space $Y$.

Theorem 2. Assume that $M(t)$ is an arbitrary Orlicz function. Let $\lambda=\left\{\lambda_{k}\right\}_{k=1}^{\infty}$ be an arbitrary sequence of the positive numbers for which the condition (4) holds. Then for any $n \in \mathbb{N}$,

$d_{\delta_{n-1}}\left(T: l_{M} \rightarrow l_{M}\right)=d_{\delta_{n-1}+1}\left(T: l_{M} \rightarrow l_{M}\right)=\ldots=d_{\delta_{n-1}}\left(T: l_{M} \rightarrow l_{M}\right)=E_{n}\left(T: l_{M} \rightarrow l_{M}\right)=\varepsilon_{n}$

where $\delta_{s}$ and $\varepsilon_{s}, s=1,2, \ldots$, are elements of the characteristic sequences $\delta(\lambda)$ and $\varepsilon(\lambda)$ of the sequence $\lambda$ and $\delta_{0}=0$.

Proof. First, let $n>1$. The dimension of the subspace $\Phi_{n-1}^{\lambda}$ of the polynomials

$$
\Phi_{n-1}=\sum_{k \in g_{n-1}^{\lambda}} a_{k} e_{k}
$$

is equal to $\delta_{n-1}$. Therefore, taking into account (10), we find

$$
\varepsilon_{n}=E_{g_{n-1}^{\lambda}}\left(T: l_{M} \rightarrow l_{M}\right) \geq d_{\delta_{n-1}}\left(T: l_{M} \rightarrow l_{M}\right) \geq d_{\delta_{n-2}}\left(T: l_{M} \rightarrow l_{M}\right) \geq \ldots \geq d_{\delta_{n}-1}\left(T: l_{M} \rightarrow l_{M}\right)
$$

Hence, to prove equality (11), it remains to show that

$$
d_{\delta_{n}-1}\left(T: l_{M} \rightarrow l_{M}\right) \geq \varepsilon_{n}, \quad n=1,2, \ldots
$$

For this, we use the well-known theorem on the diameter of the ball (see, e.g., Sec. 10.2 in [15]). According to this theorem, if the set $\mathfrak{M}$ of the normed linear space $\mathscr{X}$ with the norm $\|\cdot\|_{\mathscr{X}}$ contains a ball $\gamma U_{\nu+1}$ of radius $\gamma$ from a certain $(\nu+1)$-dimensional subspace $M_{\nu+1}$ of $\mathscr{X}$, that is, if

$$
\mathfrak{M} \supset \gamma U_{\nu+1}=\left\{y: y \in M_{\nu+1},\|y\|_{\mathscr{X}} \leq \gamma\right\}
$$


then

$$
d_{\nu}(\mathfrak{M})_{\mathscr{X}}=\inf _{F_{\nu} \in G_{\nu}} \sup _{f \in \mathfrak{M}} \inf _{u \in F_{\nu}}\|f-u\|_{\mathscr{X}} \geq \gamma
$$

where $G_{\nu}$ is the set of all $\nu$-dimensional subspaces of $\mathscr{X}$.

Let $\varepsilon_{n} B_{n, \Phi}^{\lambda}$ be the intersection of a ball of radius $\varepsilon_{n}$ in $l_{M}$ with the space $\Phi_{n}^{\lambda}$ (of dimension $\delta_{n}$ ) of polynomials of the form (12), i.e.,

$$
\varepsilon_{n} U_{n, \Phi}^{\lambda}=\left\{\Phi_{n} \in \Phi_{n}^{(\lambda)}:\left\|\Phi_{n}\right\|_{l_{M}} \leq \varepsilon_{n}\right\}
$$

Then, taking into account (99), (14) and monotonicity of the function $M(t)$, for any polynomial $\Phi_{n}=\sum_{k \in g_{n}^{\lambda}} a_{k} e_{k} \in \varepsilon_{n} B_{n, \Phi}^{\lambda}$, we have

$$
\sum_{k \in g_{n}^{\psi}} M\left(\left|a_{k}\right| / \lambda_{k}\right) \leq \sum_{k \in g_{n}^{\psi}} M\left(\left|a_{k}\right| / \varepsilon_{n}\right) \leq 1
$$

It follows that $\Phi_{n}$ is the image of some element from the unit ball $B l_{M}$. Thus, the ball $\varepsilon_{n} B_{n, \Phi}^{\lambda}$ of $\delta_{n}$-dimensional subspace $\Phi_{n}(\lambda)$ of $l_{M}$ is contained in the set $T\left(B l_{M}\right)$ of the images of all elements of the unit ball $B l_{M}$ by the action of the operator $T$. This yields relation (13) by virtue of the theorem indicated above. So, in the case $n>1$, Theorem 2 is proved. For $n=1$, the proof remains the same if we assume that $\Phi_{0}(\lambda)$ consists of the zero element $\theta=(0,0, \ldots)$ and its dimension is equal to zero.

Note that in the case, where $M(t) \equiv N(t) \equiv t^{p}, p \in[1, \infty)$, assertions of Theorem 2 and Corollary 2 follow respectively from theorems 1 and 2 of the paper [12 (see also [16, Ch. 11] Th. 3.1, 3.2). In the case of the finite dimensional spaces $l_{p}^{d}$, the assertion, similar to the assertion of Theorem 2 follows from Theorem 2.1 of Chapter VI of the monograph [17.

3. Best n-terms approximation. Following S.B. Stechkin [18, for any sequence $x \in l_{M}$, consider the quantity $\sigma_{n}\left(x, l_{M}\right)$ of its best $n$-terms approximation, which is given by the relation

$$
\sigma_{n}\left(x, l_{M}\right):=\inf _{\gamma_{n}} E_{\gamma_{n}}\left(x, l_{M}\right)=\inf _{a_{i}, \gamma_{n}}\left\|x-P_{\gamma_{n}}\right\|_{l_{M}}=\inf _{a_{i}, \gamma_{n}}\left\|x-\sum_{i \in \gamma_{n}} a_{i} e_{i}\right\|_{l_{M}}
$$

where $\gamma_{n}$ are the arbitrary collections of $n$ positive integers and $a_{i}$ are the arbitrary real numbers.

By virtue of (요), for any $x \in l_{M}$, we have

$$
\sigma_{n}\left(x, l_{M}\right)=\inf _{\gamma_{n}} \mathscr{E}_{\gamma_{n}}\left(x, l_{M}\right)=\inf _{\gamma_{n}}\left\|x-S_{\gamma_{n}}(x)\right\|_{l_{M}}=\inf _{\gamma_{n}} \inf \left\{\alpha>0: \sum_{k \notin \gamma_{n}} M\left(\left|x_{k}\right| / \alpha\right) \leq 1\right\} .
$$

The following Theorem 3 gives the exact values of the quantities

$$
\sigma_{n}\left(T: l_{p} \rightarrow l_{M}\right):=\sup _{x \in B l_{p}} \sigma_{n}\left(T x, l_{M}\right)
$$

of the best $n$-term approximation in the space $l_{M}$ of the set $T\left(B l_{p}\right)$ of the images of all the elements of the unit ball $B l_{p}$ by the action of the operator $T$. 
Obviously, the values of $\sigma_{n}\left(T: l_{p} \rightarrow l_{M}\right)$ are independent of the rearrangements of the vectors $\left\{e_{i}\right\}_{i=1}^{\infty}$. Therefore, in what follows, without losing generality, we assume that $\lambda=\left\{\lambda_{k}\right\}_{k=1}^{\infty}$ is an arbitrary nonincreasing sequence of the positive numbers, satisfying condition (4).

Theorem 3. Assume that $0<p<\infty$ is an arbitrary positive number and $M(t)$ is the Orlicz function such that $M\left(t^{1 / p}\right)$ is also the Orlicz function. Let also $\lambda=\left\{\lambda_{k}\right\}_{k=1}^{\infty}$ be an arbitrary nonincreasing sequence of the positive numbers, satisfying condition (44). Then for any $n \in \mathbb{N}$, the following equality is true:

$$
\sigma_{n}\left(T: l_{p} \rightarrow l_{M}\right)=\sup _{s>n} \frac{\left(\sum_{k=1}^{s} \lambda_{k}^{-p}\right)^{-\frac{1}{p}}}{M^{-1}\left(\frac{1}{s-n}\right)},
$$

where $M^{-1}$ is the inverse function of $M$. The least upper bound on the right-hand side of (17) is attained at some finite value $s^{*}$ of $s$. The least upper bound on the right-hand side of (16) is realized by the sequence $x^{*}=\left\{x_{k}^{*}\right\}_{k=1}^{\infty}$ given by

$$
x_{k}^{*}=\left\{\begin{array}{lc}
\left(\lambda_{k}^{p} \sum_{k=1}^{s^{*}} \lambda_{k}^{-p}\right)^{-\frac{1}{p}}, & k=1,2, \ldots, s^{*}, \\
0, & k>s^{*}
\end{array}\right.
$$

Note that in the case, where $M(t)=t^{q}$, for all $0<p, q<\infty$, similar assertions follow from theorems 5.1 and 6.1 of monograph [16, Ch. XI] (see also [13, Theorems 4.7 and 4.8] and [11, Theorem 4]. In the case of the finite dimensional spaces $l_{p}^{d}$, for all $0<p, q \leq \infty$, the quantities $\sigma_{n}\left(T: l_{p}^{d} \rightarrow l_{q}^{d}\right)$ were obtained in [14].

In proof of this assertion, we use the scheme and some of the methods proposed in [11] and [19]. First, we give some auxiliary facts.

Proposition 1. Let $M(t), t \geq 0$, be an arbitrary increasing convex down function, $M(0) \leq 0$. Then, for all numbers $t_{2}>t_{1} \geq 0$ and $A \geq B>0$, the following inequality is true:

$$
M\left(A t_{1}\right)+M\left(B t_{2}\right) \leq M\left(A\left(t_{1}+t_{2}\right)\right)
$$

Indeed, since the function $M(t)$ is convex and $M(0) \leq 0$, then for any numbers $\mu \in[0,1]$ and $t \geq 0$,

$$
M(\mu t)=M(\mu t+(1-\mu) \cdot 0) \leq \mu M(t)+(1-\mu) M(0) \leq \mu M(t)
$$

From this we have

$$
\begin{gathered}
M\left(t_{1}\right)+M\left(t_{2}\right)=M\left(\left(t_{1}+t_{2}\right) \frac{t_{1}}{t_{1}+t_{2}}\right)+M\left(\left(t_{1}+t_{2}\right) \frac{t_{2}}{t_{1}+t_{2}}\right) \leq \\
\frac{t_{1}}{t_{1}+t_{2}} M\left(t_{1}+t_{2}\right)+\frac{t_{2}}{t_{1}+t_{2}} M\left(t_{1}+t_{2}\right)=M\left(t_{1}+t_{2}\right) .
\end{gathered}
$$

Hence, given the increase of the function $M(x)$ and the inequality $A\left(t_{1}+t_{2}\right) \geq A t_{1}+B t_{2}$, we get (19):

$$
M\left(A\left(t_{1}+t_{2}\right)\right) \geq M\left(A t_{1}+B t_{2}\right) \geq M\left(A t_{1}\right)+M\left(B t_{2}\right)
$$


Putting $\mu=u / t, u \leq t$, in inequality (20), we obtain

$$
\frac{M(u)}{u} \leq \frac{M(t)}{t}, \quad u \leq t
$$

This implies that the function $M(t) / t, t>0$, doesn't decrease.

It should be noted that if the conditions of Theorem 3 are satisfied, then by virtue of monotonicity of the function $M(t) / t^{p}$, for any sequence $x \in B l_{p}$ and for arbitrary positive number $\mu$, we have $M\left(\mu\left|x_{k}\right|\right) /\left(\mu\left|x_{k}\right|\right)^{p} \leq M(\mu) / \mu^{p}$. Putting $\mu=\lambda^{*}=\max _{k \in \mathbb{N}} \lambda_{k}$, we get

$$
\sum_{k=1}^{\infty} M\left(\lambda_{k}\left|x_{k}\right|\right) \leq \sum_{k=1}^{\infty} M\left(\lambda^{*}\left|x_{k}\right|\right) \leq M\left(\lambda^{*}\right) \sum_{k=1}^{\infty}\left|x_{k}\right|^{p} \leq M\left(\lambda^{*}\right)<\infty .
$$

Hence, in this case, $T x \in l_{M}$ and the quantities $\sigma_{n}\left(T: l_{p} \rightarrow l_{M}\right)$ are well-defined.

We also need the following statement, which was established in [20] (see also [21]):

Lemma A. Let $N(t), t \geq 0$, be a convex down function, $a=\left\{a_{k}\right\}_{k=1}^{l}, b=\left\{b_{k}\right\}_{k=1}^{l}$ and $p=\left\{p_{k}\right\}_{k=1}^{l}$, $l \in \mathbb{N}$, be the sequences of the nonnegative numbers such that $a_{1} \geq a_{2} \geq \ldots a_{l}$ and $p_{k}>0$. Then

$$
\sum_{k=1}^{l} p_{k} b_{k} N\left(a_{k}\right) \leq \max _{s \in[1, l]}\left\{N\left(\frac{\sum_{k=1}^{l} p_{k} a_{k}}{\sum_{k=1}^{s} p_{k}}\right) \sum_{k=1}^{s} p_{k} b_{k}\right\} \text {. }
$$

Proof of Theorem 3. In the proof, we restrict the set of sequences $x \in B l_{p}$ on which it suffices to consider the upper bounds of the quantities $\sigma_{n}\left(T x, l_{M}\right)$ in order to obtain the estimate for the quantity $\sigma_{n}\left(T: l_{p} \rightarrow l_{M}\right)$. For this, we show that for any sequence $x$ from the set $B l_{p}$ (or from a certain subset $B \subset B l_{p}$ ), there exists the other sequence $y \in B l_{p}$ (or $y \in B$ ) (which satisfies some additional conditions) such that $\sigma_{n}\left(T x, l_{M}\right) \leq \sigma_{n}\left(T y, l_{M}\right)$.

First, denote by $B_{p}^{\prime}$ the set of all the sequences $x \in B l_{p}$ of the nonnegative numbers such that $\|x\|_{l_{p}}=1$. Obviously, in finding the exact upper bound on the right-hand side of (16), it is sufficient to consider the set $B_{p}^{\prime}$.

Indeed, for any sequence $x \in B l_{p}, x \neq \theta$, considering the sequence $x_{k}^{\prime}=\left|x_{k}\right| /\|x\|_{l_{p}}$, we see that $\left\|x^{\prime}\right\|_{l_{p}}=1$, as well as for any $k \in \mathbb{N}$, the inequality $\left|x_{k}\right| \leq x_{k}^{\prime}$ holds. Therefore, $\sigma_{n}\left(T x, l_{M}\right) \leq$ $\sigma_{n}\left(T x^{\prime}, l_{M}\right)$.

Further, denote by $B_{p}^{\prime \prime}$ the set of all the sequences $x \in B_{p}^{\prime}$ such that $\lambda_{1} x_{1} \geq \lambda_{2} x_{2} \geq \ldots$ and $x_{k}=0$ for all $k$, larger then a certain number $k_{x}$. Let us ascertain that in finding the exact upper bound on the right-hand side of (16), it is sufficient to consider the set $B_{p}^{\prime \prime}$.

Really, for any sequence $x \in B_{p}^{\prime}$, consider the sequence $x_{k}^{\prime \prime}=\frac{\overline{\lambda_{k} x_{k}}}{\lambda_{k}}$, where $\overline{\lambda_{k} x_{k}}$ is the nonincreasing rearrangement of the sequence of the numbers $\lambda_{k} x_{k}$ (since $\lambda_{k} x_{k} \rightarrow 0$ as $k \rightarrow \infty$, then this rearrangement exists). It is obvious that $\sigma_{n}\left(T x, l_{M}\right)=\sigma_{n}\left(T x^{\prime \prime}, l_{M}\right)$, and on the basis of theorem 368 of monographs [22], we get

$$
\left\|x^{\prime \prime}\right\|_{l_{p}}^{p}=\sum_{k=1}^{\infty}\left(\overline{\lambda_{k} x_{k}}\right)^{p} \leq \sum_{k=1}^{\infty} x_{k}^{p}=1
$$


hence, $x^{\prime \prime} \in B_{p}^{\prime \prime}$.

According to the definition of the set $B_{p}^{\prime \prime}$, for any sequence $x \in B_{p}^{\prime \prime}$ and any $\alpha>0$, we have $\sup _{\gamma_{n}} \sum_{k \in \gamma_{n}} M\left(\lambda_{k} x_{k} / \alpha\right)=\sum_{k=1}^{n} M\left(\lambda_{k} x_{k} / \alpha\right)$, and therefore,

$$
\sigma_{n}\left(T x, l_{M}\right)=\inf \left\{\alpha>0: \sum_{k=n+1}^{\infty} M\left(\lambda_{k} x_{k} / \alpha\right) \leq 1\right\} .
$$

This yields that in finding of the values $\sigma_{n}\left(T x, l_{M}\right)$ it is sufficient to consider only the sequences $x \in B_{p}^{\prime \prime}$ such that $\lambda_{k} x_{k}$ are equal for all $k \in[1, n+1]$.

Now, let $\mathcal{M}$ denotes the set of all the sequences $m=\left\{m_{k}\right\}_{k=1}^{\infty}$ of the nonnegative numbers $m_{k}$ such that $|m|:=\sum_{k=1}^{\infty} m_{k}=1$, the sequence $\lambda_{k} m_{k}^{\frac{1}{p}}$ does not increase and for all $k \in[1, n+1]$, the numbers $\lambda_{k} m_{k}^{\frac{1}{p}}$ are equal. Then obviously

$$
\sigma_{n}\left(T: l_{p} \rightarrow l_{M}\right)=\sup _{m \in \mathcal{M}} \inf \left\{\alpha>0: \sum_{k=n+1}^{\infty} M\left(\lambda_{k} m_{k}^{\frac{1}{p}} / \alpha\right) \leq 1\right\}=: \sup _{m \in \mathcal{M}} \mathscr{E}_{n}(m)=: \mathscr{E}_{n} .
$$

Further, denote by $\mathcal{M}^{\prime}$ the set of all the sequences $m \in \mathcal{M}$, for each of which exists the number $k_{m}$, that for all $k>k_{m}$, we have $m_{k}=0$. Show that to estimate the value $\mathscr{E}_{n}$ it suffices to consider the set $\mathcal{M}^{\prime}$.

Indeed, if the given sequence $m \in \mathcal{M}$ does not belong to the set $\mathcal{M}^{\prime}$, then there exists a number $k_{1}$, what $\lambda_{n+1} m_{n+1}^{\frac{1}{p}}>\lambda_{k_{1}} m_{k_{1}}^{\frac{1}{p}}$, and since $\sum_{k=1}^{\infty} m_{k}=1$, then there exists a number $k_{m}$, that

$$
\lambda_{n+1} m_{n+1}^{\frac{1}{p}}>\lambda_{k_{1}}\left(m_{k_{1}}+\sum_{k=k_{m}+1}^{\infty} m_{k}\right)^{\frac{1}{p}} .
$$

Consider the sequence $m^{\prime}=\left\{m_{k}^{\prime}\right\}_{k=1}^{\infty}$ such that

$$
m_{k}^{\prime}=\left\{\begin{array}{lc}
m_{k}, & k \in\left[1, k_{1}-1\right] \cup\left[k_{1}+1, k_{l}\right], \\
m_{k_{1}}+\sum_{k=k_{m}+1}^{\infty} m_{k}, & k=k_{1}, \\
0, & k>k_{l} .
\end{array}\right.
$$

Then obviously $\left|m^{\prime}\right|=|m|=1, m^{\prime} \in \mathcal{M}^{\prime}$ and due to Proposition 1 we have $\mathscr{E}_{n}(m) \leq \mathscr{E}_{n}\left(m^{\prime}\right)$. Hence,

$$
\mathscr{E}_{n}=\sup _{m \in \mathcal{M}^{\prime}} \mathscr{E}_{n}(m)=\sup _{m \in \mathcal{M}^{\prime}} \inf \left\{\alpha>0: F_{n}(m, \alpha) \leq 1\right\}
$$

where

$$
F_{n}(m, \alpha):=F_{n}(m, \lambda, \alpha, M)=\sum_{k=n+1}^{k_{m}} M\left(\lambda_{k} m_{k}^{\frac{1}{p}} / \alpha\right)
$$

Let us set $N(t)=M\left(t^{\frac{1}{p}}\right), p_{k}=\lambda_{k}^{-p}, a_{k}=\lambda_{k}^{p} m_{k} / \alpha^{p}$, where $k=1,2, \ldots, k_{m}$, and choose the numbers $b_{k}$ such that $b_{k}=0$, when $k=1,2, \ldots, n$ and $b_{k}=\lambda_{k}^{-p}$, when $k=n+1, \ldots, k_{m}$. Then we can use Lemma $\mathrm{A}$ for the estimation of the value $F_{n}(m, \alpha)$. We get

$$
F_{n}(m, \alpha)=\sum_{k=1}^{k_{m}} p_{k} b_{k} N\left(a_{k}\right) \leq \max _{s \in\left[1, k_{m}\right]}\left\{N\left(\frac{\sum_{k=1}^{k_{m}} p_{k} a_{k}}{\sum_{k=1}^{s} p_{k}}\right) \sum_{k=1}^{s} p_{k} b_{k}\right\}=
$$


$=\max _{s \in\left[1, k_{m}\right]}\left\{N\left(\frac{\sum_{k=1}^{k_{m}} m_{k}}{\alpha^{p} \sum_{k=1}^{s} \lambda_{k}^{-p}}\right)(s-n)\right\} \leq \sup _{s>n}(s-n) M\left(\tilde{\lambda}_{s} / \alpha\right)$, where $\quad \tilde{\lambda}_{s}:=\left(\sum_{k=1}^{s} \lambda_{k}^{-p}\right)^{-\frac{1}{p}}$.

Since the numbers $\lambda_{k}$ tend monotonically to zero at $k \rightarrow \infty$, then in view of (20) and the monotonicity of the function $M\left(t^{1 / p}\right) / t$, for any fixed $\alpha>0$, we obtain

$$
\lim _{s \rightarrow \infty}(s-n) M\left(\widetilde{\lambda}_{s} / \alpha\right)=\lim _{s \rightarrow \infty}(s-n) M\left(\frac{1}{\alpha}\left(\sum_{k=1}^{s} \lambda_{k}^{-p}\right)^{-\frac{1}{p}}\right) \leq K \lim _{s \rightarrow \infty} \lambda_{[s / 2]} \frac{M\left(1 / s^{1 / p}\right)}{1 / s}=0 .
$$

It follows that for any fixed $\alpha>0$, there exists at least one such natural number $s_{\alpha}$, that

$$
\sup _{s>n}(s-n) M\left(\widetilde{\lambda}_{s} / \alpha\right)=\left(s_{\alpha}-n\right) M\left(\widetilde{\lambda}_{s_{\alpha}} / \alpha\right) .
$$

To conclude the proof, for any sequence $m \in \mathcal{M}^{\prime}$, put

$$
\alpha_{m}:=\inf \left\{\alpha>0: F_{n}(m, \alpha) \leq 1\right\}
$$

and consider the sequence $\bar{m}=\left\{\bar{m}_{k}\right\}_{k=1}^{\infty}$ such that

$$
\bar{m}_{k}=\left\{\begin{array}{lc}
\widetilde{\lambda}_{s}^{p} \lambda_{k}^{-p}, & k=1,2, \ldots, s, \\
0, & k>s
\end{array}\right.
$$

where $s=s_{\alpha_{m}}$, the number $s_{\alpha_{m}}$ is determined by relation (23) at $\alpha=\alpha_{m}$, and the numbers $\tilde{\lambda}_{s}$ are defined in (21). Then it is obvious that $\bar{m} \in \mathcal{M}^{\prime}$, and by virtue of (21), we have $F_{n}\left(m, \alpha_{m}\right) \leq$ $F_{n}\left(\bar{m}, \alpha_{m}\right)$. Therefore, $\mathscr{E}_{n}(m) \leq \mathscr{E}_{n}(\bar{m})$.

Finally, denote by $\overline{\mathcal{M}}$ the set of all the sequences $\bar{m} \in \mathcal{M}^{\prime}$ of form (24), where $s, s>n$ are the arbitrary positive numbers. Then the following equality is true:

$$
\mathscr{E}_{n}=\sup _{m \in \overline{\mathcal{M}}} \mathscr{E}_{n}(m)
$$

On the basis of (24) and (25), we obtain

$$
\mathscr{E}_{n}=\sup _{s>n} \inf \left\{\alpha>0:(s-n) M\left(\widetilde{\lambda}_{s} / \alpha\right) \leq 1\right\}=\sup _{s>n} \xi_{s}
$$

where

$$
\xi_{s}:=\frac{\left(\sum_{k=1}^{s} \lambda_{k}^{-p}\right)^{-\frac{1}{p}}}{M^{-1}\left(\frac{1}{s-n}\right)},
$$

$M^{-1}$ is the inverse function of $M$. In this case, due to (22), for any $\varepsilon>0$, there exists a number $s_{\varepsilon}$ that for all $s>s_{\varepsilon}$, the value $(s-n) M\left(\tilde{\lambda}_{s} / \varepsilon\right)<1$ and therefore for all $s>s_{\varepsilon}, \xi_{s}<\varepsilon$. Hence, there exists a number $s^{*}$ such that $\sup \xi_{s}=\xi_{s^{*}}$.

Consider the sequence $x^{*}=\left\{x_{k}^{*}\right\}_{k=1}^{\infty}$ of the kind as (18). It is easy to see that $x^{*} \in B l_{p}$ and that

$$
\sigma_{n}\left(T x^{*}, l_{M}\right)=\inf \left\{\alpha>0:\left(s^{*}-n\right) M\left(\left(\sum_{k=1}^{s^{*}} \lambda_{k}^{-p}\right)^{-\frac{1}{p}} / \alpha\right)\right\}=\xi_{s^{*}}
$$


The theorem is proved.

\section{ACKNOWLEDGMENTS}

This work was supported in part by the FP7-People-2011-IRSES project number 295164 (EUMLS: EUUkrainian Mathematicians for Life Sciences).

\section{REFERENCES}

1. W. Orlicz (1936). Über Räume $\left(L^{M}\right)$. Bull. Int. Acad. Polon. Sci. A: 93-107.

2. P. B. Djakov and M. S. Ramanujan (2000). Multipliers between Orlicz Sequence Spaces. Turk. J. Math. 24: 313-319.

3. W. Orlicz (1932). Über eine gewisse Klasse von Räumen vom Typus B, Bull. Int. Acad. Polon. Sci. A: 207-220.

4. A. Zygmund (1935). Trigonometrical series. Warszawa, Lwow.

5. W. A. J. Luxemburg (1955). Banach functional spaces. Van Gorcum.

6. J. Lindenstrauss and L. Tzafriri (1977). Classical Banach spaces I. Springer-Verlag, Berlin, Heidelberg, New York.

7. Yu.I. Gribanov (1955). Nonlinear operators in Orlicz spaces. Kazan. Gos. Univ. Uchen. Zap. 115: 5-13.

8. Yu.I. Gribanov (1957). To the theory of spaces $l_{M}$. Kazan. Gos. Univ. Uchen. Zap. 117: 62-65.

9. J. Lindenstrauss and L. Tzafriri (1971). On Orlicz sequence spaces. Israel J. Math. 10: 379-390.

10. M. Aiyub (2013). On some seminormed sequence spaces defined by Orlicz function. Proyecciones Journal of Mathematics. 32: 267-280.

11. A. I. Stepanets (2001). Approximation characteristics of the spaces $S_{\varphi}^{p}$ in different metrics. Ukr. Math. J. 53: 1340-1374.

12. A. I. Stepanets (2001). Approximation characteristics of spaces $S_{\varphi}^{p}$. Ukr. Math. J. 53: 446-475.

13. A. I. Stepanets (2006). Problems of approximation theory in linear spaces. Ukr. Math. J. 58: 47-92.

14. Fuchang Gao (2010). Exact value of the $n$-term approximation of a diagonal operator. J. Approx. Theory 162: 646-652.

15. V. M. Tikhomirov (1976). Some Problems in Approximation Theory. Moscow University, Moscow.

16. A.I. Stepanets (2005). Methods of Approximation Theory. VSP, Leiden-Boston.

17. A. Pinkus (1985). n-widths in approximation theory. Springer-Verlag, Berlin .

18. S. B. Stechkin (1955). On the absolute convergence of orthogonal series Dokl. Akad. Nauk SSSR. 102: 37-40.

19. A. I. Stepanets and A.L. Shydlich (2005). On one extremal problem for positive series. Ukr. Mat. Zh. 57: 1677-1683.

20. A. L. Shidlich and S. O. Chaichenko (2014). On some inequalities of Chebyshev type [in press].

21. A.L. Shidlich and S.O. Chaichenko (2014). On some inequalities of Chebyshev type. arXiv preprint, arXiv:1405.1256v1.

22. G. H. Hardy, J. E. Littlewood, and G. Pólya (1934). Inequalities. Cambridge University Press, Cambridge. 